\title{
Research Article \\ High Order Differential Frequency Hopping: Design and Analysis
}

\author{
Yong Li, ${ }^{1,2}$ Fuqiang Yao, ${ }^{2}$ and $\mathrm{Ba} \mathrm{Xu}^{2}$ \\ ${ }^{1}$ Institute of Communications Engineering, PLA University of Science and Technology, Nanjing 210007, China \\ ${ }^{2}$ Nanjing Telecommunication Technology Institute, Nanjing 210007, China \\ Correspondence should be addressed to Yong Li; liy771121@163.com
}

Received 28 November 2014; Accepted 9 June 2015

Academic Editor: Marco Mussetta

Copyright (c) 2015 Yong Li et al. This is an open access article distributed under the Creative Commons Attribution License, which permits unrestricted use, distribution, and reproduction in any medium, provided the original work is properly cited.

\begin{abstract}
This paper considers spectrally efficient differential frequency hopping (DFH) system design. Relying on time-frequency diversity over large spectrum and high speed frequency hopping, DFH systems are robust against hostile jamming interference. However, the spectral efficiency of conventional DFH systems is very low due to only using the frequency of each channel. To improve the system capacity, in this paper, we propose an innovative high order differential frequency hopping (HODFH) scheme. Unlike in traditional DFH where the message is carried by the frequency relationship between the adjacent hops using one order differential coding, in HODFH, the message is carried by the frequency and phase relationship using two-order or higher order differential coding. As a result, system efficiency is increased significantly since the additional information transmission is achieved by the higher order differential coding at no extra cost on either bandwidth or power. Quantitative performance analysis on the proposed scheme demonstrates that transmission through the frequency and phase relationship using two-order or higher order differential coding essentially introduces another dimension to the signal space, and the corresponding coding gain can increase the system efficiency.
\end{abstract}

\section{Introduction}

As one of the two basic modulation techniques used in spread spectrum communications, frequency hopping $(\mathrm{FH})$ was originally designed to be inherently secure and reliable under adverse battle conditions for military applications. However, there are three major bottlenecks: low data rate, narrow bandwidth frequency hopping, and a low hopping rate when using frequency hopping in the high frequency (HF) band. Currently, the data rate of the existing HF frequency hopping radio is limited to $2400 \mathrm{bps}$ or less, the reliable data rate is $1200 \mathrm{bps}$, the highest hopping rate is only tens of hops per sec, and the antijamming capability is weak. Under current conditions, it is difficult to achieve high-capacity communications with HF communications technology when there are harsh electromagnetic environments.

In the 1990s, the Sanders Company, from the United States of America, pioneered an enhanced hopping spread spectrum radio (Correlated Hopping Enhanced Spread Spectrum), referred to as CHESS Radio $[1,2]$. It was based on the differential frequency hopping system and was a good solution to improve the data rate and anti-tracking-jamming abilities, among other issues. Its hopping rate is 5000 hops per sec and the frequency hopping bandwidth is $2 \mathrm{MHz}$; these were breakthroughs compared to conventional HF communication systems. However, the conventional DFH system has low spectral efficiency over large bandwidth. Typically, conventional DFH systems require large bandwidth, which is proportional to the requirement on the frequency number. Along with the ever increasing demand on inherently secure high data rate wireless communications, new techniques that are more efficient and reliable have to be developed. In literature [3], a FH system called modulated differential frequency hopping was proposed, in this system, some information was used to produce a pseudo-random carrier frequency slot (as in the DFH technology), and others were changed into narrowband modulated waveforms (as in the conventional FH technology). The message-driven frequency hopping $(\mathrm{MDFH})$ scheme proposed in literature [4] possesses higher spectral efficiency than conventional $\mathrm{FH}$ 
communication system. In literature [5], based on the idea of $\mathrm{MDFH}$, a new scheme was proposed to improve the ability of anti-fixed-frequency interference. In literature $[6,7]$, the antijamming performance of the MDFH was analyzed in depth.

In this paper, we propose an innovative high order differential frequency hopping $(\mathrm{HODFH})$ scheme. The basic idea is that the frequencies and the phases of the signal are numbered and then using the two-order or higher order differential coding to code the message and the signal number. The message is parsed into several parts; each part will be transmitted by different order differential coding. In other words, the serial message stream is converted into parallel data stream transmitted by each order differential coding. At the receiver, the transmitting frequency is captured using a filter bank as in the FSK receiver rather than using the frequency synthesizer. As a result, the frequency can be blindly detected at each hop. This relaxes the burden of strict frequency synchronization at the receiver. And the phase is estimated as in the PSK receiver. Then the frequency and the phase are used to decode the message. The system spectral efficiency is significantly improved, because the high order differential coding and the phases do not require extra cost on bandwidth and power.

In this paper, in-depth performance analysis is conducted based on both theoretical derivation and simulation examples. Our analysis demonstrates that phases essentially add another dimension to the signal space, and the high order differential coding can increase the system spectral efficiency.

\section{The Concept of High Order Differential Frequency Hopping}

2.1. Transmitter Design. Unlike the conventional DFH systems in which only the frequencies are coded by the oneorder coding, the HODFH systems code the frequencies and their phases by high order coding. That is to say, in the conventional DFH system, only the carrier frequencies carry the message; however, in the HODFH system, not only frequencies but also the phases carry the information. In a HODFH system, we add several phases to the frequencies; therefore, the signal number is significantly increased almost without increase in bandwidth.

Let $N_{c}$ be the total number of frequencies, with $\left\{f_{1}, f_{2}, \ldots, f_{N_{c}}\right\}$ being the set of all frequencies. Let $M_{k}$ be the number of initial phases where the frequency is $f_{k}$ in each hop, with $\left\{\phi_{1}, \phi_{2}, \ldots, \phi_{M_{k}}\right\}$ being the set of all phases. Define $s\left(f_{n, i}, \phi_{n, j}\right)$ as the combinated signal of these frequencies and phases in the $n$th hop; it is clear that there are $N_{s}=\sum_{n=1}^{N_{c}} M_{k}$ signals. The set $\mathbf{S}$, with $\left\{s_{1}, s_{2}, \ldots, s_{N_{s}}\right\}$ being its elements, is the set of all the combinated signals.

Let $N_{o}$ be the order of the HODFH system; we start by reshaping the one-dimensional set $S$ into $N_{o}$-dimensional set $\mathbf{S}_{D_{1} \times D_{2} \times \cdots \times D_{N_{o}}}$, where the subscript denotes element number of each dimension, and $D_{1} \times D_{2} \times \cdots \times D_{N_{o}} \leq N_{s}$; denote $s_{d_{1}, d_{2}, \ldots, d_{N_{o}}}$ as the element of the set $\mathbf{S}_{D_{1} \times D_{2} \times \cdots \times D_{N_{o}}}$. We assume that $D_{1} \times D_{2} \times \cdots \times D_{N_{o}}=N_{s}$. Divide the

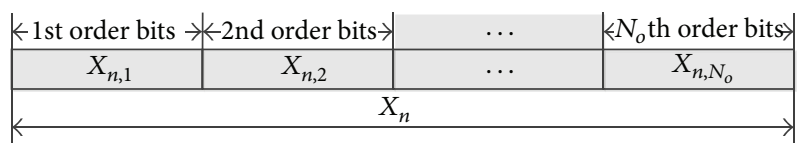

FIGURE 1: The $n$th block of the information data.

data stream into blocks of length $L \triangleq \sum_{i=1}^{N_{o}} B_{i}$, where $B_{i}(\leq$ $\left.\log _{2} D_{i}\right)$ is the number of bits that is transmitted through the $i$ th order differential coding. Denote the $n$th block by $X_{n}$. The block $X_{n}$ can be grouped into $N_{o}$ vectors, denoted by $\left[X_{n, 1}, X_{n, 2}, \ldots, X_{n, N_{o}}\right]$, where $X_{n, i}$ denotes the $i$ th order bits, as shown in Figure 1. We will transmit $X_{n}$ within one hop.

The transmitter block diagram of the proposed $\mathrm{HODFH}$ scheme is illustrated in Figure 2. Each input data block, $X_{n}$, is fed into a serial-to-parallel $(\mathrm{S} / \mathrm{P})$ converter, where the data bits are split into $N_{o}$ parallel data streams. Denote the signals in the $n$th and $(n-1)$ th hop by $s_{d_{1}^{n}, d_{2}^{n}, \ldots, d_{N_{o}}^{n}}^{n}$ and $s_{d_{1}^{n-1}, d_{2}^{n-1}, \ldots, d_{N_{o}}^{n-1}}^{n-1}$ The index of the $s_{d_{1}^{n}, d_{2}^{n}, \ldots, d_{N_{o}}^{n}}^{n}$ is obtained by $N_{o^{-}}$ order differential coding the index of the $s_{d_{1}^{n-1}, d_{2}^{n-1}, \ldots, d_{N_{o}}^{n-1}}^{n-1}$ and information data block $X_{n}$. The differential processing is called a function $G$ and expressed by the following equations:

$$
\begin{aligned}
& d_{1}^{n}=G_{1}\left(d_{1}^{n-1}, d_{2}^{n-1}, \ldots, d_{N_{o}}^{n-1}, X_{n, 1}\right) \\
& d_{m}^{n}=G_{m}\left(d_{m-1}^{n}, X_{n, m}\right), \quad \text { when } m=2, \ldots, N_{o} .
\end{aligned}
$$

Using the index vectors to select the combinated signal, the signal transmitted over the channel is $s_{d_{1}^{n}, d_{2}^{n}, \ldots, d_{N_{0}}^{n}}^{n}$.

Recall that $s\left(f_{n, i}, \phi_{n, j}\right)$ is the combination of the frequency and phase. The transmitted signal in the $n$th hop can be expressed as

$$
\begin{aligned}
& s_{d_{1}^{n}, d_{2}^{n}, \ldots, d_{N_{o}}^{n}}^{n}=s\left(f_{n, i}, \phi_{n, j}\right)=A \cos \left(2 \pi f_{n, i} t+\right.\left.\phi_{n, j}\right) \\
& 0 \leq t<T_{h},
\end{aligned}
$$

where $A$ is the signal amplitude, $f_{n, i}$ is the center frequency of the $n$th hop, $\phi_{n, j}$ is the possible phases of the $n$th hop, and $T_{h}$ is the hop duration. The passband waveform of the transmitted signal may be expressed as

$$
s(t)=\sqrt{\frac{2}{T_{h}}} \operatorname{Re}\left\{\sum_{n=-\infty}^{\infty} e^{-j 2 \pi\left(f_{n, i}+\phi_{n, j}\right) t} g\left(t-n T_{h}\right)\right\},
$$

where $g(t)$ is the signal pulse-shaping filter.

2.2. Receiver Design. The receiver block diagram of the proposed HODFH scheme is illustrated in Figure 3. Recall that there are $N_{c}$ frequencies $\left\{f_{1}, f_{2}, \ldots, f_{N_{c}}\right\}$ that are extended out $N_{s}$ signals. First, we must detect which frequency is transmitted. For this purpose, a bank of $N_{c}$ bandpass filters (BPF), each centered at $f_{i}\left(i=1,2, \ldots, N_{c}\right)$, with the same channel bandwidth as the transmitter, is deployed at the receiver front end. Since only one frequency band is occupied at any given hop, we simply measure the outputs of bandpass filters at each possible signaling frequency. The actual carrier 


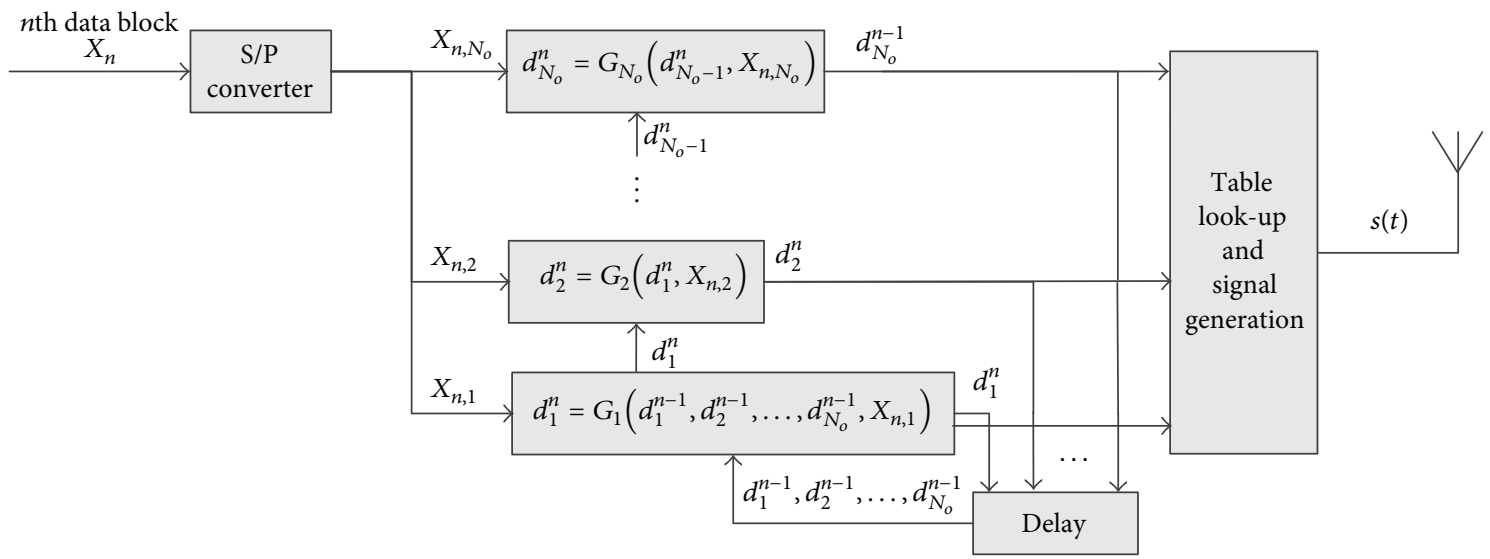

FIGURE 2: The transmitter block diagram of the proposed HODFH scheme.

frequency at a certain hopping period can be detected by selecting the one that captures the strongest signal. As a result, blind detection of the carrier frequency is achieved at the receiver.

More specifically, the received signal is a convolution of the transmitted signal $s(t)$ with the channel impulse response, and it can be expressed as

$$
r(t)=\int_{-\infty}^{+\infty} h(\tau) s(t-\tau) d \tau+n(t)
$$

where $h(\tau)$ is the channel impulse response and $n(t)$ denotes additive white Gaussian noise (AWGN). Accordingly, the outputs of bandpass filters are given by

$$
z_{i}(t)=\int_{-\infty}^{+\infty} q_{i}(\tau) r(t-\tau) d \tau, \quad \text { for } i=1, \ldots, N_{c},
$$

where $q_{i}(t)$ is the ideal bandpass filter centered at frequency $f_{i}$. If the channel is ideal, that is, $h(t)=\delta(t)$, then

$$
z_{i}(t)=\int_{-\infty}^{+\infty} q_{i}(\tau) s(t-\tau) d \tau+u_{i}(t)
$$

$$
\text { for } i=1, \ldots, N_{c} \text {, }
$$

where $u_{i}(t)=\int_{-\infty}^{+\infty} q_{i}(\tau) n(t-\tau)$ is the filtered version of WGN. If the signal-to-noise ratio is sufficiently high, and there is no strong jamming, as in most communication systems, there is one and only one significantly strong signal among the outputs of the filter bank. Suppose that the $l$ th filter captures this distinctive signal during the $n$th hop; then the estimated hopping frequency $\hat{f}_{n, i}=f_{l}$. The same procedures can be carried out to determine the carrier frequency at each hop.

Next, the estimated hopping frequency $\widehat{f}_{n, i}$ is used to control NCO. The output signal multiplied by the received signal and NCO is used to estimate the phase of the transmitted signal. Denote the estimated phase as $\widehat{\phi}_{n, j}$.

Then, the estimated hopping frequency $\widehat{f}_{n, i}$ and the estimated phase $\widehat{\phi}_{n, j}$ are used to estimate the transmitted signal. Denote the estimated signal as $s_{\widehat{d}_{1}^{n}, \widehat{d}_{2}^{n}, \ldots, \hat{d}_{N_{o}}^{n}}$. The estimated index $\left\{\widehat{d}_{1}^{n}, \ldots, \widehat{d}_{N_{o}}^{n}\right\}$ at the $n$th hop and the estimated index $\left\{\widehat{d}_{1}^{n-1}, \ldots, \widehat{d}_{N_{o}}^{n-1}\right\}$ at the $(n-1)$ th hop are fed into the function $G_{m}^{-1}\left(1 \leq m \leq N_{o}\right)$, the inverse function of $G_{m}$, to recover the information data blocks. This processing can be expressed by the following equations:

$$
\begin{aligned}
& \widehat{X}_{n, 1}=G_{1}^{-1}\left(\widehat{d}_{1}^{n-1}, \widehat{d}_{2}^{n-1}, \ldots, \widehat{d}_{N_{o}}^{n-1}, \widehat{d}_{1}^{n}\right), \\
& \widehat{X}_{n, m}=G_{m}^{-1}\left(\widehat{d}_{m-1}^{n}, \widehat{d}_{m}^{n}\right), \quad \text { when } m=2, \ldots, N_{o} .
\end{aligned}
$$

It then follows that the estimate of the $n$th block $X_{n}$ can be obtained as $\widehat{X}_{n}=\left[\widehat{X}_{n, 1}, \widehat{X}_{n, 2}, \ldots, \widehat{X}_{n, N_{o}}\right]$.

\section{Bit Error Probability for HODFH}

In HODFH system, the input bit stream is carried by hopping frequency and its phase. So, firstly, we analyze hopping frequency symbol error probability and phase symbol error probability, respectively, and then deduce the bit error rate (BER) of the HODFH.

3.1. Symbol Error Probability. Firstly, we analyze the hopping frequency symbol error probability. Based on the receiver design in $\mathrm{HODFH}$, analysis of the hopping frequency symbol error probability is analogous to that of noncoherent FSK demodulation. For noncoherent detection of $M_{F}$-ary FSK signals, the probability of symbol error is given by $[8$, eqn. (5-4-46), page 310]:

$$
\begin{aligned}
& p_{s, \mathrm{FSK}}\left(\frac{\varepsilon_{s}}{N_{0}}\right) \\
& =\sum_{n=1}^{M_{F}-1}(-1)^{n+1}\left(\begin{array}{c}
M_{F}-1 \\
n
\end{array}\right) \frac{1}{n+1} \exp \left[-\frac{n \varepsilon_{s}}{(n+1) N_{0}}\right],
\end{aligned}
$$

where $\varepsilon_{s} / N_{0}$ is the SNR per symbol. For a HODFH system with $N_{c}$ frequencies, $M_{F}=N_{c}$. Let $p_{s \text {, HODFH }}^{(F)}$ denote the probability of the hopping frequency symbol detection error 


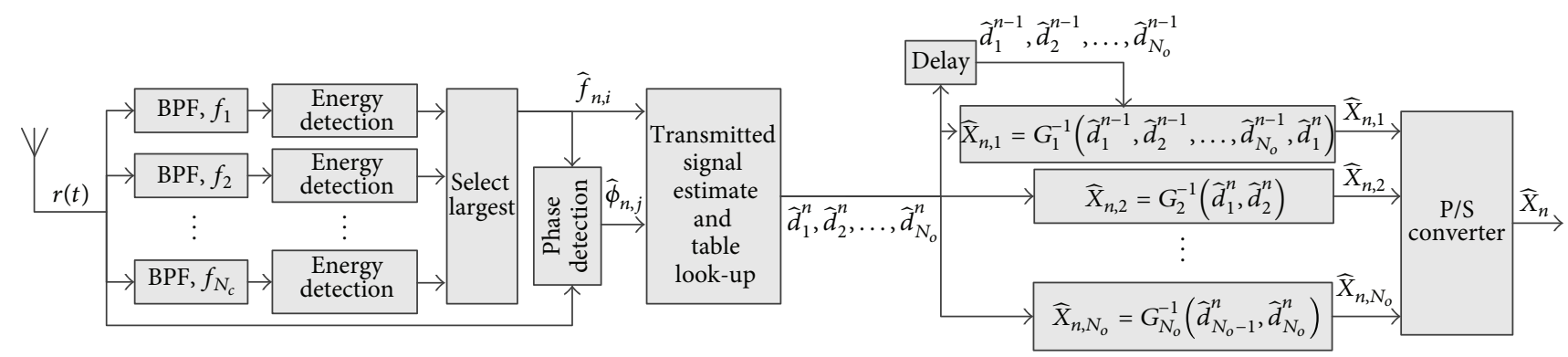

FIgURE 3: The receiver block diagram of the proposed HODFH scheme.

(corresponding to the symbol error in FSK) in HODFH; then we have

$$
\begin{aligned}
& p_{s, \mathrm{HODFH}}^{(F)}\left(\frac{\varepsilon_{s}^{F}}{N_{0}}\right) \\
& \quad=\sum_{n=1}^{N_{c}-1}(-1)^{n+1}\left(\begin{array}{c}
N_{c}-1 \\
n
\end{array}\right) \frac{1}{n+1} \exp \left[-\frac{n \varepsilon_{s}^{F}}{(n+1) N_{0}}\right],
\end{aligned}
$$

where $\varepsilon_{s}^{F} / N_{0}$ is the SNR of hopping frequency per symbol.

Next, we focus on the phase symbol error probability. The phase symbol error probability can be calculated in a similar manner as that of the $M_{P}$-ary PSK. The probability of phase symbol error probability is given by [8, eqn. (5-2-56) \& (5-255), page 268]:

$$
\begin{aligned}
& p_{s, \mathrm{PSK}}\left(\frac{\varepsilon_{s}}{N_{0}}\right)=1-\int_{-\pi / M_{P}}^{\pi / M_{P}} p_{\Theta_{r}}\left(\Theta_{r}\right) d \Theta_{r}, \\
& p_{\Theta_{r}}\left(\Theta_{r}\right)=\frac{1}{2 \pi} \exp \left(-\frac{\varepsilon_{s}}{N_{0}} \sin ^{2} \Theta_{r}\right) \\
& . \int_{0}^{\infty} V \exp \left[-\frac{\left(V-\sqrt{2\left(\varepsilon_{s} / N_{0}\right)} \cos \Theta_{r}\right)^{2}}{2}\right] d V .
\end{aligned}
$$

For a HODFH system, signal phase detection is implemented at the condition that the hopping frequency is detected correctly, for which the probability is $\left(1-p_{s, \mathrm{HODFH}}^{(F)}\right)$; the probability of phase symbol error can be calculated as that of the $M_{P}$-ary PSK. Let $p_{s, \mathrm{HODFH}}^{(P)}$ denote the probability of the phase symbol detection error (corresponding to the symbol error in PSK). Recall that there are $M_{k}$ possible phases in each hopping frequency; we have

$$
p_{s, \mathrm{HODFH}}^{(P)}\left(\frac{\varepsilon_{s}^{P}}{N_{0}}\right)=1-\int_{-\pi / M_{k}}^{\pi / M_{k}} p_{\Theta_{r}}\left(\Theta_{r}\right) d \Theta_{r},
$$

where $\varepsilon_{s}^{P} / N_{0}$ is the SNR of phase per symbol and it will be substituted in (11) when we calculate $p_{s, \mathrm{HODFH}}^{(P)}$.

Only when the hopping frequency and the phase are detected correctly, the transmitted signal can be estimated correctly. Since, in a HODFH system, the transmitted signal carries the frequency and phase information, the SNR of the hopping frequency per symbol is the same as the SNR of the phase; that is, $\varepsilon_{s}^{F} / N_{0}=\varepsilon_{s}^{P} / N_{0}$. We denote the SNR per symbol by $\varepsilon_{s} / N_{0}$. The overall symbol error probability of the HODFH scheme is calculated as the combination of $p_{s, \mathrm{HODFH}}^{(F)}$ and $p_{s, \mathrm{HODFH}}^{(P)}$, defined as $p_{s, \mathrm{HODFH}}$, which can be found in

$$
\begin{aligned}
p_{s, \mathrm{HODFH}}\left(\frac{\varepsilon_{s}}{N_{0}}\right) \\
=1 \\
\quad-\left(1-p_{s, \mathrm{HODFH}}^{(F)}\left(\frac{\varepsilon_{s}}{N_{0}}\right)\right)\left(1-p_{s, \mathrm{HODFH}}^{(P)}\left(\frac{\varepsilon_{s}}{N_{0}}\right)\right) \\
=p_{s, \mathrm{HODFH}}^{(F)}\left(\frac{\varepsilon_{s}}{N_{0}}\right)+p_{s, \mathrm{HODFH}}^{(P)}\left(\frac{\varepsilon_{s}}{N_{0}}\right) \\
\quad-p_{s, \mathrm{HODFH}}^{(F)}\left(\frac{\varepsilon_{s}}{N_{0}}\right) \cdot p_{s, \mathrm{HODFH}}^{(P)}\left(\frac{\varepsilon_{s}}{N_{0}}\right) .
\end{aligned}
$$

3.2. Bit Error Probability. It is reasonable to assume that the probability of the index $\widehat{d}_{i}^{n}\left\{1 \leq i \leq N_{o}\right\}$ estimated incorrectly is inversely proportionate to the number of elements at the $i$ th dimensionality, when the symbol error occurs. Let $p_{\text {Index }, i}$ be the error probability of the $i$ th dimensional index; we have

$$
p_{\text {Index }, i}\left(\frac{\varepsilon_{s}}{N_{0}}\right)=\frac{D_{i}-1}{D_{i}} p_{s, \mathrm{HODFH}}\left(\frac{\varepsilon_{s}}{N_{0}}\right) .
$$

For the first order bits $X_{n, 1}$, it is only decided by $\left\{\widehat{d}_{1}^{n-1}, \ldots, \widehat{d}_{N_{o}}^{n-1}\right\}$ and $\widehat{d}_{1}^{n}$. The correct probability of $\widehat{d}_{1}^{n-1}, \ldots, \widehat{d}_{N_{o}}^{n-1}$ is equal to the symbol correct probability $\left(1-p_{s, \mathrm{HODFH}}\right)$. Recall that there are $B_{1}$ bits at the first order. The BER of the first order bits, denoted as $p_{b 1, \mathrm{HODFH}}$, is obtained:

$$
\begin{aligned}
p_{b 1, \mathrm{HODFH}}\left(\frac{\varepsilon_{s}}{N_{0}}\right)=\frac{2^{B_{1}-1}}{2^{B_{1}}-1}(1 \\
\left.-\left(1-p_{\text {Index }, 1}\left(\frac{\varepsilon_{s}}{N_{0}}\right)\right)\left(1-p_{s, \mathrm{HODFH}}\left(\frac{\varepsilon_{s}}{N_{0}}\right)\right)\right) \\
=\frac{2^{B_{1}-1}}{2^{B_{1}}-1}\left(\frac{2 D_{1}-1}{D_{1}} p_{s, \mathrm{HODFH}}\left(\frac{\varepsilon_{s}}{N_{0}}\right)\right. \\
\left.-\frac{D_{1}-1}{D_{1}}\left(p_{s, \mathrm{HODFH}}\left(\frac{\varepsilon_{s}}{N_{0}}\right)\right)^{2}\right) .
\end{aligned}
$$


For the $m$ th order bits $X_{n, m}\left(2 \leq m \leq N_{o}\right)$, it is decided by $\widehat{d}_{m-1}^{n}$ and $\widehat{d}_{m}^{n}$. Only the $\widehat{d}_{m-1}^{n}$ and $\widehat{d}_{m}^{n}$ are estimated correctly; the information bits can be recovered correctly. Assume that the error probability of $\widehat{d}_{m-1}^{n}$ and $\widehat{d}_{m}^{n}$ is independent. This is insured by the processing of reshaping the 1-dimensional set $S$ into $N_{o}$-dimensional set $S_{D_{1} \times D_{2} \times \cdots \times D_{N_{o}}}$. Let $p_{\text {Index }}^{i, j}$ be the correct probability of the $i$ th and the $j$ th dimensional index simultaneity; we have

$$
\begin{aligned}
& p_{\text {Index }}^{i, j}\left(\frac{\varepsilon_{s}}{N_{0}}\right)=\left(1-p_{\text {Index }, i}\left(\frac{\varepsilon_{s}}{N_{0}}\right)\right) \\
& \cdot\left(1-p_{\text {Index }, j}\left(\frac{\varepsilon_{s}}{N_{0}}\right)\right) \\
& =\left(1-\frac{D_{i}-1}{D_{i}} p_{s, \mathrm{HODFH}}\left(\frac{\varepsilon_{s}}{N_{0}}\right)\right) \\
& \cdot\left(1-\frac{D_{j}-1}{D_{j}} p_{s, \mathrm{HODFH}}\left(\frac{\varepsilon_{s}}{N_{0}}\right)\right)=1-\frac{D_{j}-1}{D_{j}} \\
& \cdot p_{s, \mathrm{HODFH}}\left(\frac{\varepsilon_{s}}{N_{0}}\right)-\frac{D_{i}-1}{D_{i}} p_{s, \mathrm{HODFH}}\left(\frac{\varepsilon_{s}}{N_{0}}\right) \\
& +\frac{\left(D_{i}-1\right)\left(D_{j}-1\right)}{D_{i} D_{j}}\left(p_{s, \mathrm{HODFH}}\left(\frac{\varepsilon_{s}}{N_{0}}\right)\right)^{2} .
\end{aligned}
$$

Recall that there are $B_{m}$ bits at the $m$ th order. The BER of the $m$ th order bits, denoted as $p_{b m, \mathrm{HODFH}}$, is obtained:

$$
\begin{aligned}
& p_{b m, \mathrm{HODFH}}=\frac{2^{B_{m}-1}}{2^{B_{m}}-1}\left(1-p_{\text {Index }}^{m-1, m}\right) \\
& =\frac{2^{B_{m}-1}}{2^{B_{m}}-1}\left(\frac{D_{m-1}-1}{D_{m-1}} p_{s, \mathrm{HODFH}}\left(\frac{\varepsilon_{s}}{N_{0}}\right)\right. \\
& +\frac{D_{m}-1}{D_{m}} p_{s, \mathrm{HODFH}}\left(\frac{\varepsilon_{s}}{N_{0}}\right) \\
& \left.-\frac{\left(D_{m-1}-1\right)\left(D_{m}-1\right)}{D_{m-1} D_{m}}\left(p_{s, \mathrm{HODFH}}\left(\frac{\varepsilon_{s}}{N_{0}}\right)\right)^{2}\right) .
\end{aligned}
$$

Therefore, the total BER of a HODFH system, denoted as $p_{b, \mathrm{HODFH}}$, can be obtained as

$$
\begin{aligned}
p_{b, \mathrm{HODFH}}\left(\frac{\varepsilon_{s}}{N_{0}}\right) & \\
= & \left(\frac{B_{1}}{\sum_{n=1}^{N_{o}} B_{n}}\right) \cdot p_{b 1, \mathrm{HODFH}}\left(\frac{\varepsilon_{s}}{N_{0}}\right) \\
& +\sum_{m=2}^{N_{o}}\left(\frac{B_{m}}{\sum_{n=1}^{N_{o}} B_{n}}\right) \cdot p_{b m, \mathrm{HODFH}}\left(\frac{\varepsilon_{s}}{N_{0}}\right) .
\end{aligned}
$$

Since $\sum_{n=1}^{N_{o}} B_{n}=L,(18)$ can be transformed as

$$
\begin{gathered}
p_{b, \mathrm{HODFH}}\left(\frac{\varepsilon_{s}}{N_{0}}\right)=\frac{1}{L}\left(B_{1} p_{b 1, \mathrm{HODFH}}\left(\frac{\varepsilon_{s}}{N_{0}}\right)\right. \\
\left.+\sum_{m=2}^{N_{o}} B_{m} p_{b m, \mathrm{HODFH}}\left(\frac{\varepsilon_{s}}{N_{0}}\right)\right) .
\end{gathered}
$$

We assume that one symbol not correctly detected will incur the $N_{o}$ dimensional index being not correctly estimated. That is to say, $p_{\text {Index }, i}$ and $p_{\text {Index }}^{i, j}$ will satisfy the following equations:

$$
\begin{aligned}
& p_{\text {Index }, i}\left(\frac{\varepsilon_{s}}{N_{0}}\right)=p_{s, \mathrm{HODFH}}\left(\frac{\varepsilon_{s}}{N_{0}}\right) \\
& p_{\text {Index }}^{i, j}\left(\frac{\varepsilon_{s}}{N_{0}}\right) \\
& =\left(1-p_{s, \mathrm{HODFH}}\left(\frac{\varepsilon_{s}}{N_{0}}\right)\right)\left(1-p_{s, \mathrm{HODFH}}\left(\frac{\varepsilon_{s}}{N_{0}}\right)\right) \\
& =1-2 p_{s, \mathrm{HODFH}}\left(\frac{\varepsilon_{s}}{N_{0}}\right)+\left(p_{s, \mathrm{HODFH}}\left(\frac{\varepsilon_{s}}{N_{0}}\right)\right)^{2} .
\end{aligned}
$$

Substitute (20) into (15) and (17); the uniform of the upper bound of BER for the first and the $m$ th order bits, denoted as $p_{b m, \mathrm{HODFH}}^{U}$, can be obtained as

$$
\begin{aligned}
& p_{b m, \mathrm{HODFH}}^{U}=\frac{2^{B_{m}-1}}{2^{B_{m}}-1}\left(2 p_{s, \mathrm{HODFH}}\left(\frac{\varepsilon_{s}}{N_{0}}\right)\right. \\
& \left.-\left(p_{s, \mathrm{HODFH}}\left(\frac{\varepsilon_{s}}{N_{0}}\right)\right)^{2}\right), \quad 1 \leq m \leq N_{o} .
\end{aligned}
$$

When the symbol error probability $p_{s, \mathrm{HODFH}}$ is small enough, the bit error probability $p_{b m, \mathrm{HODFH}}^{U}$ can be approximated as

$$
\begin{aligned}
p_{b m, \mathrm{HODFH}}^{U}\left(\frac{\varepsilon_{s}}{N_{0}}\right)=\frac{2^{B_{m}}}{2^{B_{m}}-1} p_{s, \mathrm{HODFH}}\left(\frac{\varepsilon_{s}}{N_{0}}\right), & \\
& 1 \leq m \leq N_{o} .
\end{aligned}
$$

Substitute (22) into (19); the upper bound of total BER for the HODFH system is obtained

$$
\begin{aligned}
p_{b, \mathrm{HODFH}}^{U}\left(\frac{\varepsilon_{s}}{N_{0}}\right) & =\frac{1}{L} \sum_{m=1}^{N_{o}} B_{m} p_{b m, \mathrm{HODFH}}^{U}\left(\frac{\varepsilon_{s}}{N_{0}}\right) \\
& =\frac{1}{L} \sum_{m=1}^{N_{o}} B_{m} \frac{2^{B_{m}}}{2^{B_{m}}-1} p_{s, \mathrm{HODFH}}\left(\frac{\varepsilon_{s}}{N_{0}}\right) .
\end{aligned}
$$

The lower bound of total BER, denoted as $p_{b, \mathrm{HODFH}}^{L}$, can be obtained by assuming that the symbol error only incurs bits error in that hopping. The error probability of the $i$ th dimensional index is expressed as (14), and the 
$i$ th dimensional index error only incurs the $i$ th order bits. Therefore, the lower bound of total BER is

$$
\begin{aligned}
& p_{b, \mathrm{HODFH}}^{L}\left(\frac{\varepsilon_{s}}{N_{0}}\right) \\
& \quad=\frac{1}{L} \sum_{m=1}^{N_{o}} B_{m} \frac{2^{B_{m}-1}\left(D_{m}-1\right)}{\left(2^{B_{m}}-1\right) D_{m}} p_{s, \mathrm{HODFH}}\left(\frac{\varepsilon_{s}}{N_{0}}\right) .
\end{aligned}
$$

To summarize our discussions above, we have the following.

Proposition. In HODFH, the total BER, $p_{b, \mathrm{HODFH}}\left(\varepsilon_{s} / N_{0}\right)$, is bounded by

$$
\begin{gathered}
\frac{1}{L} \sum_{m=1}^{N_{o}} B_{m} \frac{2^{B_{m}-1}\left(D_{m}-1\right)}{\left(2^{B_{m}}-1\right) D_{m}} p_{s, \mathrm{HODFH}}\left(\frac{\varepsilon_{s}}{N_{0}}\right) \\
\quad \leq p_{b, \mathrm{HODFH}}\left(\frac{\varepsilon_{s}}{N_{0}}\right) \\
\quad \leq \frac{1}{L} \sum_{m=1}^{N_{o}} B_{m} \frac{2^{B_{m}}}{2^{B_{m}}-1} p_{s, \mathrm{HODFH}}\left(\frac{\varepsilon_{s}}{N_{0}}\right) .
\end{gathered}
$$

3.3. Further Discussions about Bit Error Probability. Recall that there are $N_{c}$ available frequencies $\left\{f_{1}, f_{2}, \ldots, f_{N_{c}}\right\}$ for the HODFH system, and they distribute in broad band. It only occupies narrow band, centered at $f_{i}\left(i=1,2, \ldots, N_{c}\right)$, available frequencies at any given moment. The SNR usually is unequal at each narrow band. In this occasion, the symbol error probability of the frequency and phase cannot be simply calculated by (9) and (12). The symbol correct decision probability $p_{c, f_{i}}$ of the frequency $f_{i}$ can be deduced by [8, eqn. (5-4-41), page 309]. Because the random variables $R_{i}(i=$ $\left.1,2, \ldots, N_{c}\right)$ are statistically independent and identically distributed and we assume that the signals centered at the $N_{c}$ available frequencies $f_{1}, f_{2}, \ldots, f_{N_{c}}$ have equal energy and different noise, the probability $p_{c, f_{i}}$ can be expressed as

$$
p_{c, f_{i}}=\int_{0}^{\infty} \prod_{\substack{m=1 \\ m \neq i}}^{N_{c}} P\left(R_{m}<\frac{\sigma_{i}}{\sigma_{m}} R_{i} \mid R_{i}=x\right) p_{R_{i}}(x) d x
$$

where $\sigma_{m}^{2}=1 / 2 N_{0, m}$ is the variance of the AWGN at frequency $f_{i}$ and

$$
\begin{aligned}
P & \left(R_{m}<\frac{\sigma_{i}}{\sigma_{m}} R_{i} \mid R_{i}=x\right) \\
& =\int_{0}^{\sigma_{i} x / \sigma_{m}} p_{R_{m}}\left(r_{m}\right) d r_{m}=1-e^{-\sigma_{i}^{2} x^{2} / 2 \sigma_{m}^{2}} \\
& =1-e^{-N_{0, i} x^{2} / 2 N_{0, m}} .
\end{aligned}
$$

Substitute (27) into (26); we obtain the general probability

$$
p_{c, f_{i}}=\int_{0}^{\infty} \prod_{\substack{m=2 \\ m \neq i}}^{N_{c}}\left(1-e^{-N_{0, i} x^{2} / 2 N_{0, m}}\right) p_{R_{i}}(x) d x .
$$

We have known that, in a HODFH system, signal phase detection is implemented at the condition that the hopping frequency centred at $f_{i}$ is detected correctly, for which the probability is $p_{c, f_{i}}$; the probability of phase symbol error can be calculated as (10) and (11), but the SNR $\varepsilon_{s}^{P} / N_{0}$ of phase per symbol will be substituted by $\varepsilon / N_{0, i}$, which is the SNR at frequency $f_{i}$. Let $p_{s, \mathrm{HODFH}}^{(P, i)}$ denote the probability of the phase symbol detection error at frequency $f_{i}$. In this occasion, the overall symbol error probability of the HODFH scheme is calculated by

$$
\begin{aligned}
p_{s, \mathrm{HODFH}}^{\prime} & =1-\sum_{i=1}^{N_{c}} p_{i} \cdot p_{c, f_{i}} \cdot\left(1-p_{s, \mathrm{HODFH}}^{(P, i)}\right) \\
& =1-\frac{1}{N_{c}} \sum_{i=1}^{N_{c}} p_{c, f_{i}} \cdot\left(1-p_{s, \mathrm{HODFH}}^{(P, i)}\right),
\end{aligned}
$$

where the $p_{i}\left(i=1,2, \ldots, N_{c}\right)$ is the probability that the signals centered at frequency $f_{i}$ and the $p_{1}, p_{2}, \ldots, p_{N_{c}}$ all equal $1 / N_{c}$. Substitute the probability $p_{s, \mathrm{HODFH}}$ by $p_{s, \mathrm{HODFH}}^{\prime}$ in (16) and (17); we can calculate the total BER of a HODFH system by (18) when it works in the occasion that the $N_{c}$ available frequencies $\left\{f_{1}, f_{2}, \ldots, f_{N_{c}}\right\}$ distribute in broad band and the SNR is not equal at each narrow band centered at $f_{i}$.

\section{Simulation Results}

Without loss of generality, in this paper, the HODFH system in the AWGN channel with equal SNR at anywhere was simulated and its bit error rate (BER) performance was analyzed.

Example 1. BER performance of the HODFH system in AWGN channel: the performance of BER at the information rates of $9600 \mathrm{bps}, 19200 \mathrm{bps}$, and $28800 \mathrm{bps}$ in AWGN channel was compared. For the information rate of $9600 \mathrm{bps}$, $N_{o}=2, N_{c}=128, M_{k}=2\left(k=1, \ldots, N_{c}\right)$, and $B_{k}=1(k=$ $\left.1, \ldots, N_{o}\right)$. For the information rate of $19200 \mathrm{bps}, N_{o}=2$, $N_{c}=64, M_{k}=4\left(k=1, \ldots, N_{c}\right)$, and $B_{k}=2\left(k=1, \ldots, N_{o}\right)$. For the information rate of $28800 \mathrm{bps}, N_{o}=2, N_{c}=64$, $M_{k}=4\left(k=1, \ldots, N_{c}\right), B_{1}=4$, and $B_{2}=2$.

Figure 4 is the BER performance of the HODFH system in AWGN channel. The requirement for the energy-per-bit noise rate $\left(E_{b} / N_{0}\right)$ becomes lower when the information rate increases. In practice, it is usually possible that only the energy-per-symbol noise rate $\left(E_{s} / N_{0}\right)$ can be measured. According to formula $E_{s} / N_{0}=E_{b} / N_{0}+10 \log (k)$ ( $k$ is the number of bits per symbol), convert $E_{b} / N_{0}$ to $E_{s} / N_{0}$, and then the higher $E_{s} / N_{0}$ is required for the higher information rate, which is reasonable.

Example 2. The BER performance of information rate 28800 bps at different number of phases: assume $N_{o}=2$ and $B_{k}=3\left(k=1, \ldots, N_{o}\right)$. The numbers of available frequencies $N_{c}$ are 64, 32, and 16; the numbers of phases $M_{k}$ are 4,8 , and 16 , respectively. 


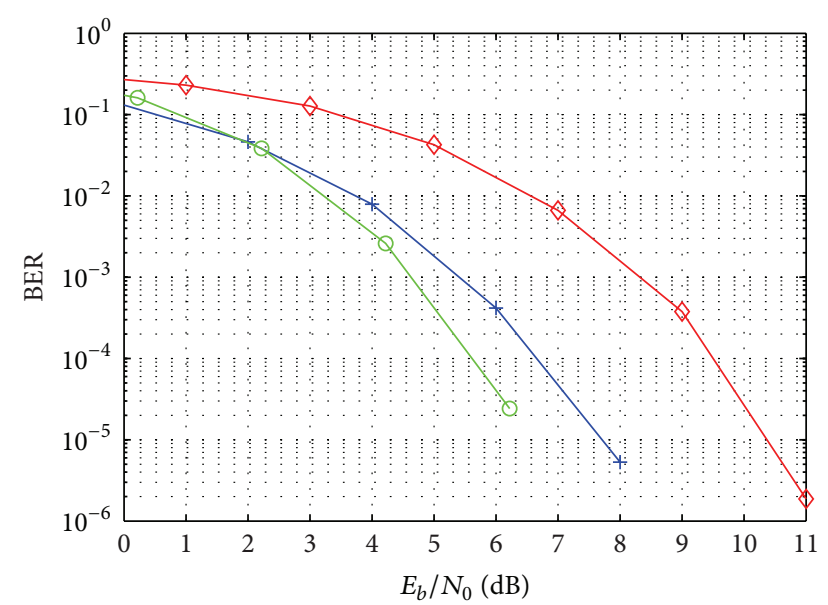

$$
\begin{aligned}
& \smile 9600 \mathrm{bps}, N_{o}=2, N_{c}=128, M_{k}=2, B_{k}=1 \\
& \leftarrow 19200 \mathrm{bps}, N_{o}=2, N_{c}=64, M_{k}=4, B_{k}=2 \\
& \odot 28800 \mathrm{bps}, N_{o}=2, N_{c}=64, M_{k}=4, B_{1}=4, B_{2}=2
\end{aligned}
$$

FIGURE 4: The BER performance at information rates of $9600 \mathrm{bps}$, 19200 bps, and 28800 bps in AWGN channel.

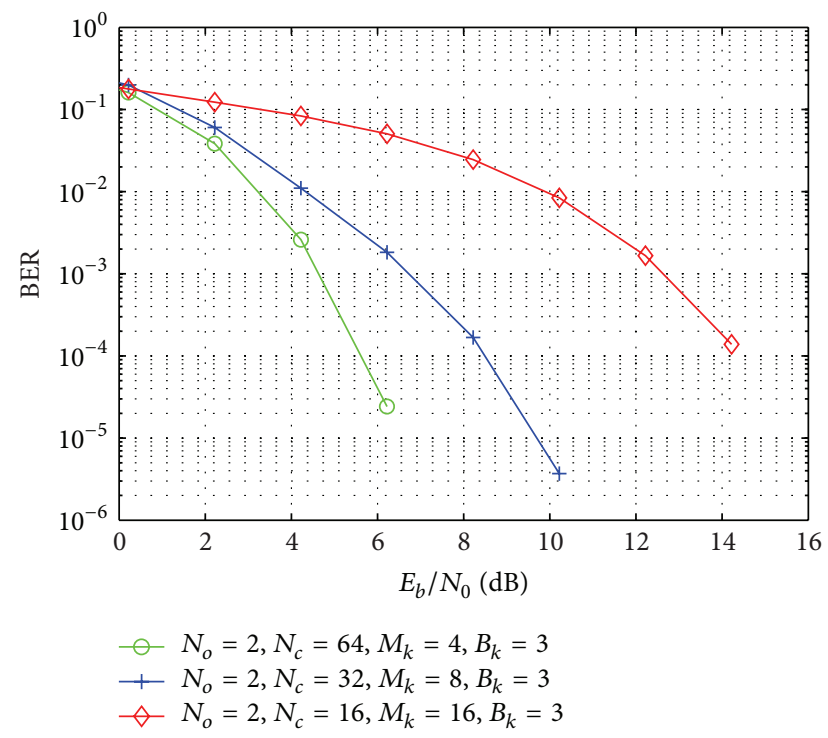

FIGURE 5: The BER performance of information rate $28800 \mathrm{bps}$ at different number of phases.

As seen in Figure 5, when more phrases are used while keeping the information rate the same, there is poorer performance for the HODFH system. It will spend about $6 \mathrm{~dB}$ extra $E_{b} / N_{0}$ to obtain the same BER performance of $10^{-3}$ when the number of phases increases from 8 to 16. Therefore, the number of phases should be kept to a minimum, if the number of transmission signals has reached the requirements.

Example 3. The BER performance of the HODFH system and the conventional DFH (CDFH) system: for the information rate of $9600 \mathrm{bps}$, the HODFH system has the following parameters: $N_{o}=2, N_{c}=128, M_{k}=2\left(k=1, \ldots, N_{c}\right)$, and

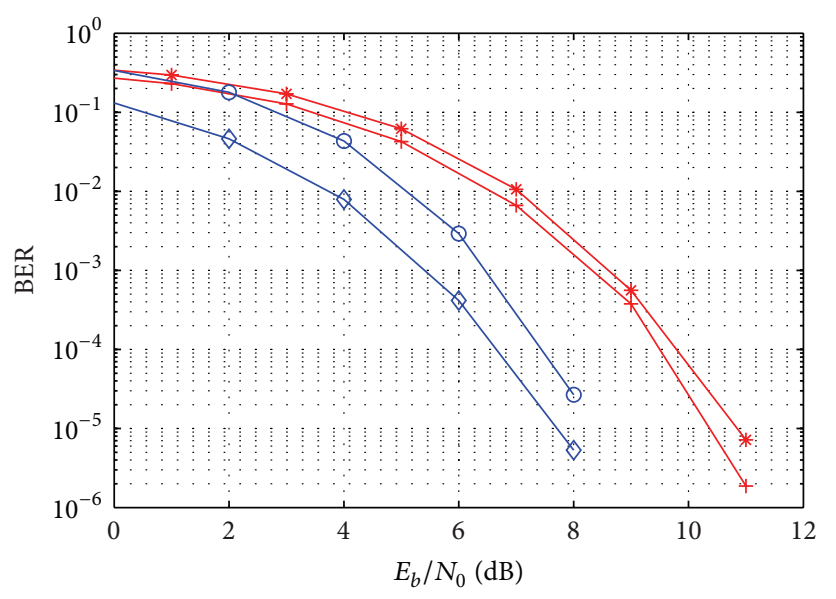

$$
\begin{aligned}
& * 9600 \mathrm{bps}, \mathrm{CDFH}, N_{c}=128, \mathrm{BPH}=2 \\
& +9600 \mathrm{bps}, \mathrm{HODFH}, N_{o}=2, N_{c}=128, M_{k}=2, B_{k}=1 \\
& \odot 19200 \mathrm{bps}, \mathrm{CDFH}, N_{c}=128, \mathrm{BPH}=4 \\
& \curvearrowright 19200 \mathrm{bps}, \mathrm{HODFH}, N_{o}=2, N_{c}=64, M_{k}=4, B_{k}=2
\end{aligned}
$$

FIGURE 6: The BER performance of the HODFH system and the CDFH system.

$B_{k}=1\left(k=1, \ldots, N_{o}\right)$; for the information rate of $19200 \mathrm{bps}$, it has parameters: $N_{o}=2, N_{c}=64, M_{k}=4\left(k=1, \ldots, N_{c}\right)$, and $B_{k}=2\left(k=1, \ldots, N_{o}\right)$. The CDFH has the following parameters: for information rate of $9600 \mathrm{bps}, N_{c}=128$ and $\mathrm{BPH}=2$; for information rate of $19200 \mathrm{bps}, N_{c}=128$ and $\mathrm{BPH}=4$.

Figure 6 shows the BER performance comparison of the HODFH system and the conventional DFH system for information rates of $9600 \mathrm{bps}$ and $19200 \mathrm{bps}$. At the same information rate, the HODFH system has a better BER performance than the conventional DFH system. The higher information rate is, the higher gain is obtained by the HODFH system when compared to the conventional DFH system.

Example 4. Compare the BER performances of the HODFH system at three information rates $(9600 \mathrm{bps}, 19200 \mathrm{bps}$, and 28800 bps) with the theoretical BER performance of QPSK, 8PSK, 16PSK, 32PSK, and 64PSK. Assume the HODFH system has the following parameters, respectively. For the information rate of $9600 \mathrm{bps}, N_{o}=2, N_{c}=128, M_{k}=2(k=$ $\left.1, \ldots, N_{c}\right)$, and $B_{k}=1\left(k=1, \ldots, N_{o}\right)$. For the information rate of $19200 \mathrm{bps}, N_{o}=2, N_{c}=64, M_{k}=4\left(k=1, \ldots, N_{c}\right)$, and $B_{k}=2\left(k=1, \ldots, N_{o}\right)$. For the information rate of 28800 bps, we have three combinations; the first is $N_{o}=2$, $N_{c}=64, M_{k}=4\left(k=1, \ldots, N_{c}\right)$, and $B_{k}=3\left(k=1, \ldots, N_{o}\right)$, the second is $N_{o}=2, N_{c}=32, M_{k}=8\left(k=1, \ldots, N_{c}\right)$, and $B_{k}=3\left(k=1, \ldots, N_{o}\right)$, and the third is $N_{o}=2, N_{c}=16$, $M_{k}=16\left(k=1, \ldots, N_{c}\right)$, and $B_{k}=3\left(k=1, \ldots, N_{o}\right)$.

Figure 7 presents the BER performance of the $\mathrm{HODFH}$ system and the theoretical BER performance of QPSK, 8PSK, 16PSK, 32PSK, and 64PSK. When the information rate is 9600 bps, the HODFH system carries two bits of information 

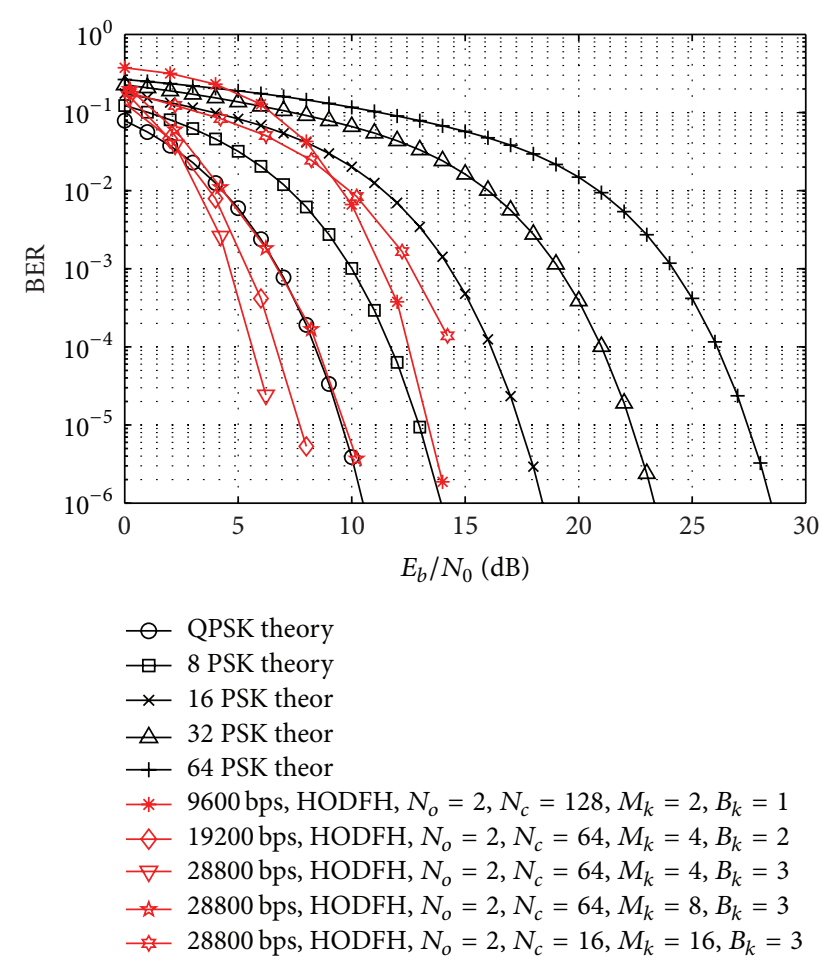

FIGURE 7: The BER performance of the HODFH system and theoretical BER performance of the M-PSK.

per hop (symbol), which is the same as the QPSK; the BER performance of the HODFH system is worse than the QPSK. When the information rate is $19200 \mathrm{bps}$, the HODFH system carries four bits of information per hop (symbol), which is the same as the 16PSK, but it has better BER performance than the 16PSK. When the information rate is $28800 \mathrm{bps}$, the HODFH system carries six bits of information per hop (symbol), which is the same as the 64PSK, but its performance is much better than the 64PSK no matter how many phases are used by the HODFH system. The BER performance of the HODFH system is better than the M-PSK when the information rate is high. The carrier frequencies of the HODFH systems are used to transmit information; the HODFH systems have higher information rate without extra cost on power and do not need extra cost on power, when the numbers of phases adopted by the HODFH systems and the M-PSK are equal. The HODFH systems have better BER performance at the same energyper-bit noise rate compared to the PSK.

Example 5. Compare the BER performance of information rate $19200 \mathrm{bps}$ at the occasion that the SNR is equal and unequal at each narrow band. Assume that the SNRs at $N_{c} / 2$ narrow bands are different with the SNRs at the other $N_{c} / 2$ narrow bands. Use $\mathrm{SNR}_{d}$ to denote the difference among the $N_{c}$ SNRs. The $\mathrm{SNR}_{d}$ increases from 2 to 8 by increment 2. Use the average SNR in the broad band as the symbol signal-to-noise ratio. The HODFH system has the following parameters: $N_{o}=2, N_{c}=64, M_{k}=4\left(k=1, \ldots, N_{c}\right)$, and $B_{k}=2\left(k=1, \ldots, N_{o}\right)$.

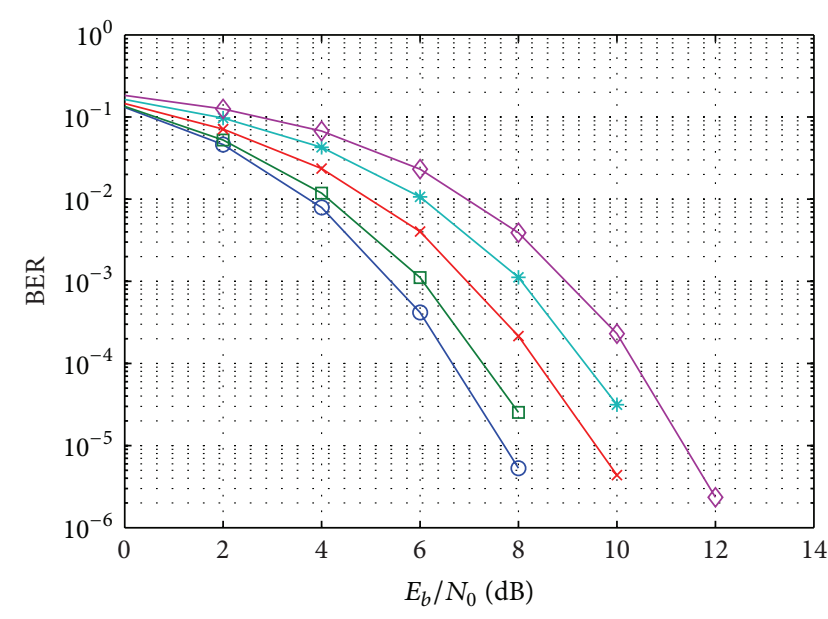

$$
\begin{aligned}
& \bigcirc \text { Equal SNR } \quad * \text { Unequal SNR, } \mathrm{SNR}_{d}=6
\end{aligned}
$$

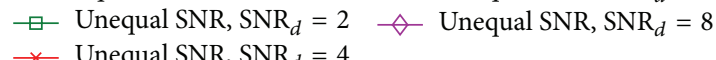

FIGURE 8: The BER performance of information rate $19200 \mathrm{bps}$ at $\mathrm{SNR}_{d}=2,4,6$, and 8 .

Example 6. Compare the BER performance of information rate 28800 bps at different number of phases when the SNR difference $\mathrm{SNR}_{d}=4$ and 6 . The SNR is similar as Example 5. The HODFH system has the following parameters: $N_{o}=2$, $N_{c}=64, B_{k}=3\left(k=1, \ldots, N_{o}\right)$, and $M_{k}=4,8$, and $16(k=$ $\left.1, \ldots, N_{c}\right)$.

As seen in Figure 8, the BER performance is worse when the SNR is unequal at each narrow band, despite the average SNR of the unequal signal-to-noise ratio broad band being the same as the SNR of the equal signal-to-noise ratio. As the difference of the SNRs is increasing, the performance is degraded.

Figure 9 shows that the BER performance is degraded when the number of phases used by the HODFH system increases. This recalls the conclusion in Example 2 that the number of phases should be kept to a minimum, if the number of transmission signals has reached the requirements.

\section{Summary}

In the future, communication will have higher information rate requirements, and this paper proposed a solution for this with a novel differential frequency hopping, high order differential frequency hopping method. This was done by conumbering the frequencies and their phases, so that the system information rate was improved without having to increase the frequency resources. The BER performance of the HODFH system is better than the conventional DFH system and M-PSK in the AWGN channel. In this work, the number of phases can be adjusted to match the channel conditions to obtain the optimal performance.

Although using phases to expand the signal set can improve the bandwidth efficiency, their introduction of the 


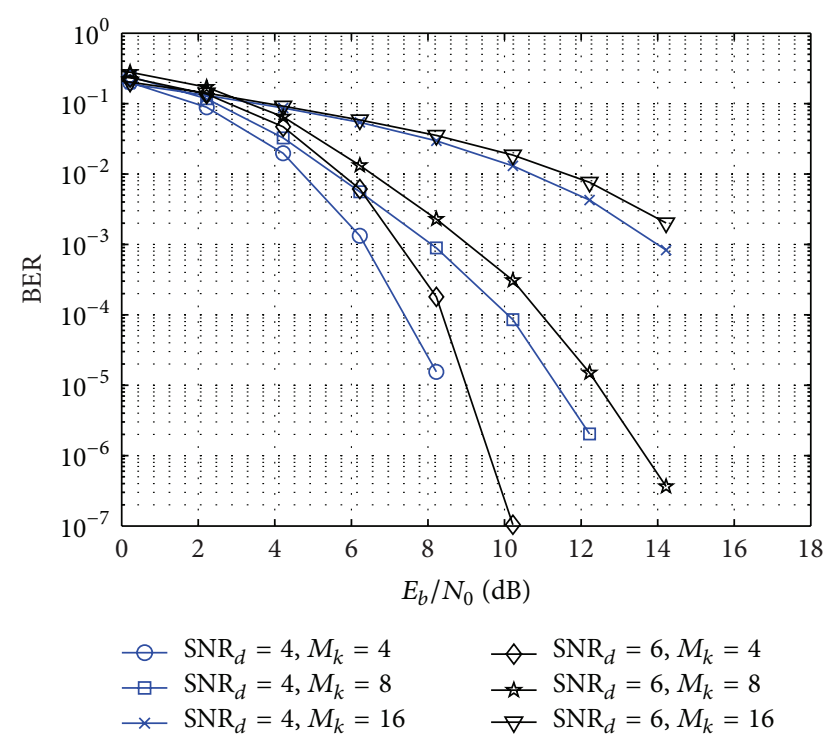

FIGURE 9: BER performance of information rate $28800 \mathrm{bps}$ at different number of phases when $\mathrm{SNR}_{d}=4$ and 6 .

phases makes the HODFH system more sensitive to frequency offset and timing errors. That is to say, implementation of this system is more complex, but it is a price worth paying for the information rate improvement and increased performance.

\section{Conflict of Interests}

The authors declare that there is no conflict of interests regarding the publication of this paper.

\section{References}

[1] D. L. Herrick and P. K. Lee, "CHESS: a new reliable high speed HF radio," in Proceedings of the IEEE Military Communications Conference (MILCOM '96), pp. 684-690, McLean, Va, USA, 1996.

[2] D. L. Herrick, P. K. Lee, and L. L. Ledlow Jr., "Correlated frequency hopping: an improved approach to HF spread spectrum communications," in Proceedings of the Tactical Communications Conference, pp. 319-324, IEEE, Fort Wayne, Ind, USA, April-May 1996.

[3] B. Yang and Y. Shen, "Modulated (soft-decision) differential frequency hopping system," in Proceedings of the International Conference on Information and Communication Technology (ICICT '07), pp. 209-212, March 2007.

[4] Q. Ling and T. Li, "Message-driven frequency hopping: design and analysis," IEEE Transactions on Wireless Communications, vol. 8, no. 4, pp. 1773-1782, 2009.

[5] D. Wang, H. Zhao, and Z. Fan, "A new scheme for messagedriven FH system," in Proceedings of the International Conference on Future Information Technology and Management Engineering (FITME '10), pp. 395-398, October 2010.

[6] L. Zhang, H. Wang, and T. Li, "Anti-jamming message-driven frequency hopping. Part I. System design," IEEE Transactions on Wireless Communications, vol. 12, no. 1, pp. 70-79, 2013.
[7] L. Zhang and T. Li, "Anti-jamming message-driven frequency hopping-Part II: capacity analysis under disguised jamming," IEEE Transactions on Wireless Communications, vol. 12, no. 1, pp. P80-P88, 2013.

[8] J. G. Proakis, Digital Communications, McGraw-Hill, 4th edition, 2001. 


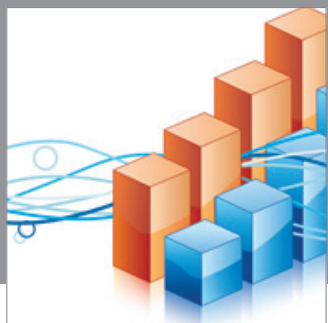

Advances in

Operations Research

mansans

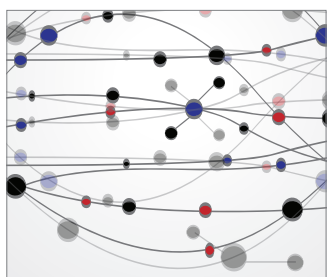

The Scientific World Journal
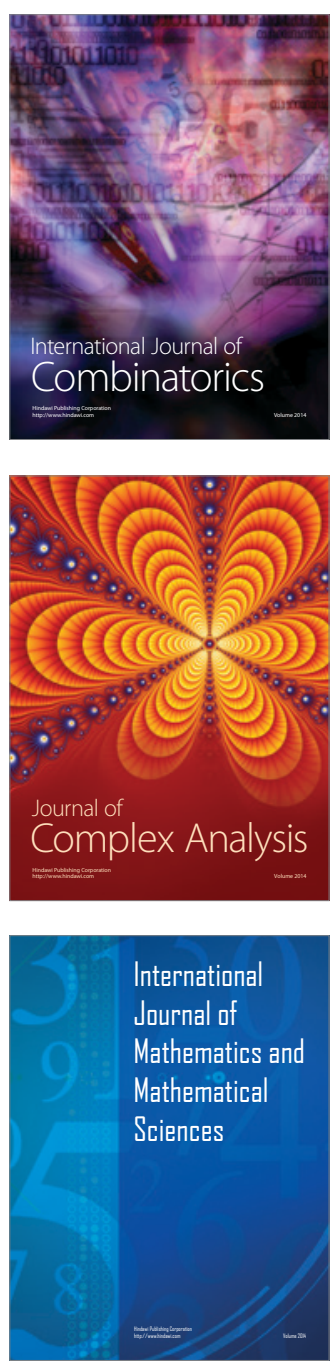
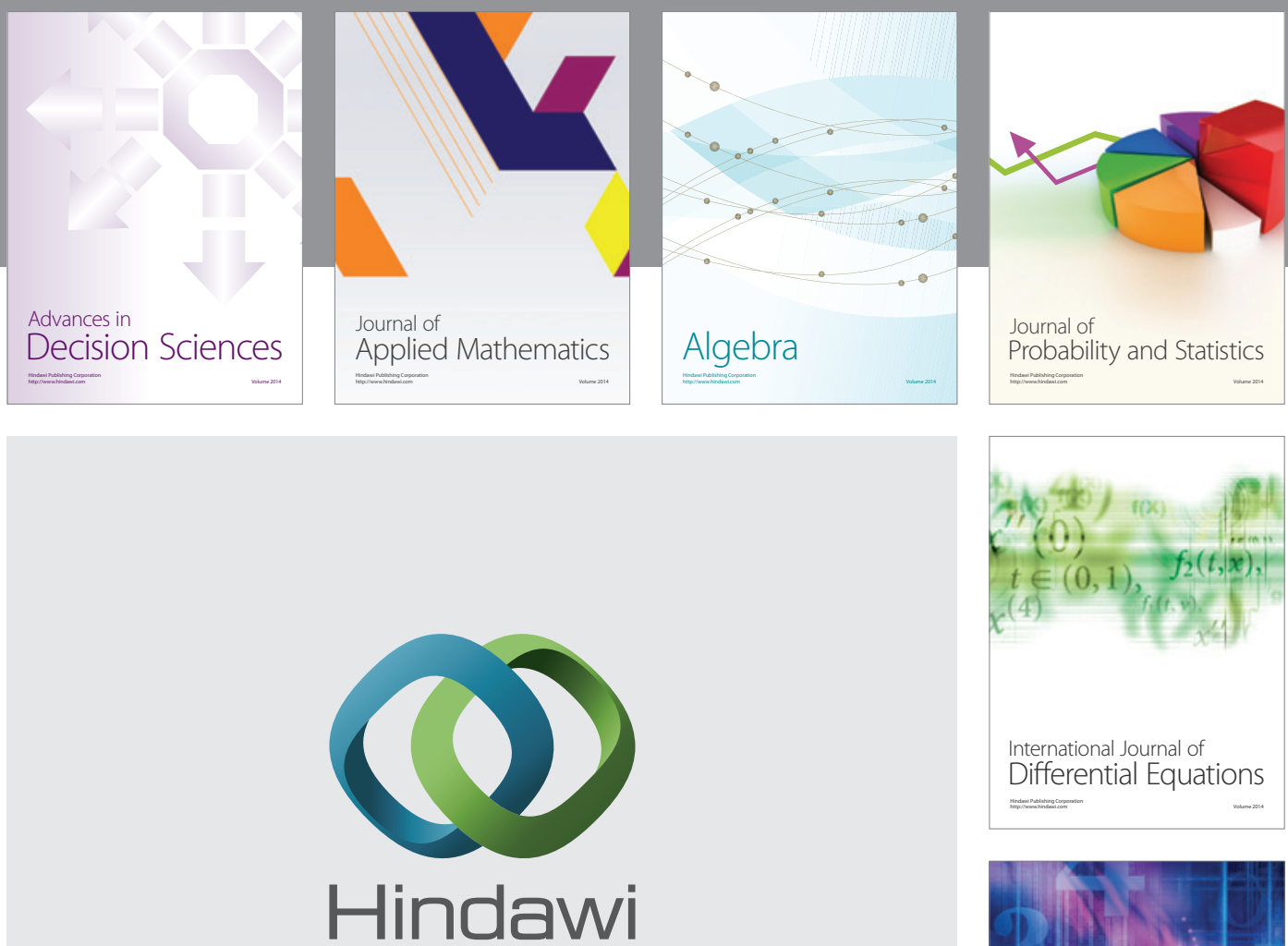

Submit your manuscripts at http://www.hindawi.com
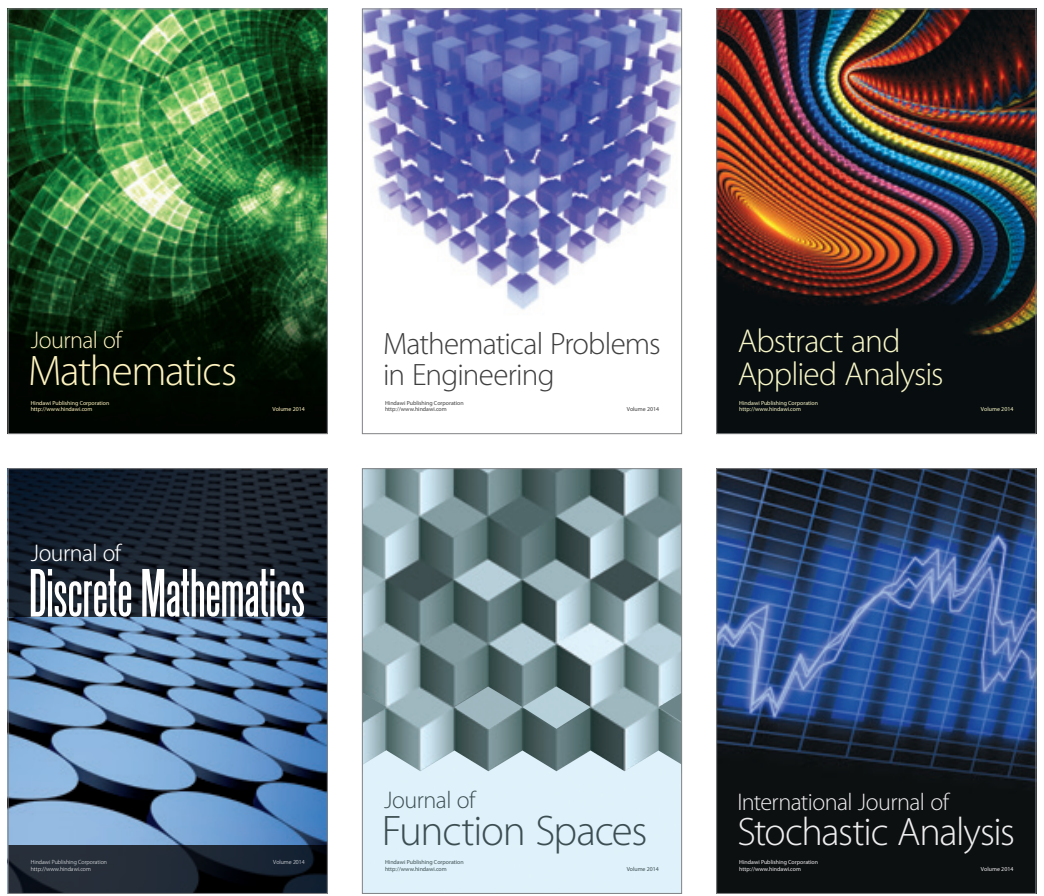

Journal of

Function Spaces

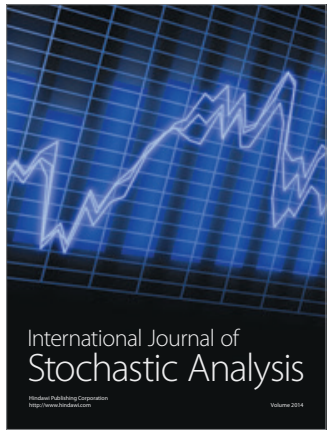

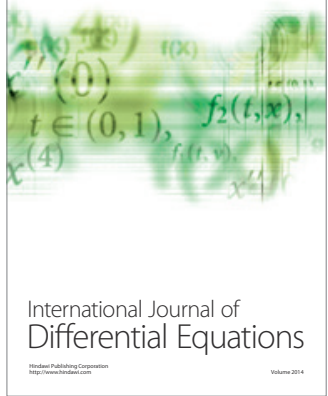
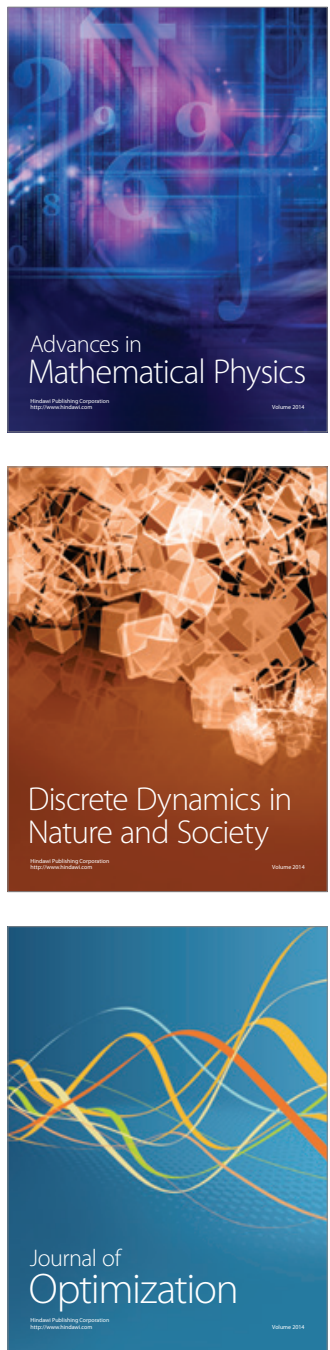